\title{
Educación financiera en los niños: una evidencia empírica
}

\section{Children financial education: An empirical evidence}

\section{Evangelina CRUz BARBA*}

La alfabetización financiera implica un proceso que proporciona herramientas necesarias para comprender e interpretar el mundo económico. Este artículo analiza los determinantes de la educación financiera de los niños de educación básica. Mediante pruebas de independencia estadística chi-cuadrada, examina los resultados de una encuesta aplicada a la población estudiantil del sexto grado de primaria en cinco escuelas públicas de la zona metropolitana de Guadalajara, Jalisco, México. La evidencia empírica identifica a la familia como la principal influencia en la alfabetización financiera de los niños. Ahorro, utilidad de los bancos y decisiones de compra son conceptos clave usados por los niños como parte del lenguaje familiar. Existen relaciones significativas entre la experiencia financiera de los niños en la vida diaria de la familia, la disposición de ellos a aprender, y la creación de una conciencia financiera para generar competencias en ese campo para su vida diaria. De acuerdo con estos resultados, implementar una educación financiera formal en las escuelas, mediante un proceso de enseñanza-aprendizaje financiero, podría promover el entendimiento del mundo económico.

Financial education involves a process that provides tools needed to understand and to interpret the economic world. In this paper, we analyze the determinants of financial education on children in basic education. Using chi square statistical independence tests, we analyze the results of a survey applied to the sixth grade primary school students in 5 public schools in the Metropolitan Area of Guadalajara, Jalisco, Mexico. Our empirical evidence identifies the family as the main influence in the children financial literacy. Saving, bank's utility and purchasing decisions are keys concepts used by children as a part of the family language. There are significant relations among the living financial experience of children in daily family practices, their willingness to learn, and the creation of financial conscience in order to generate financial competences for the everyday life. According to these results, implementing formal financial education in the school, by a financial teaching-learning process, may promote the understanding of the economic world.

\section{Palabras clave:}

educación financiera, educación básica, modelo educativo, evidencia empírica

\section{Keywords:}

financial education, basic education, education model, empirical evidence

Recibido: 19 de octubre de 2017. | Aceptado para su publicación: 16 de marzo de 2018. Recuperado de: https://sinectica.iteso.mx/index.php/SINECTICA/article/view/809 DOI: 10.31391/S2007-7033(2018)0051-012

*Doctora en Educación. Profesora en el Departamento de Ciencias Sociales y Jurídicas del Centro Universitario de Ciencias Económico Administrativas de la Universidad de Guadalajara. Sus líneas de investigación versan sobre la educación en contextos de las ciencias económico administrativas. Correo electrónico: evangelinacruz@live.com.mx 


\section{INTRODUCCIÓN}

T a integración de mercados a nivel internacional trae aparejado la transformación

institucional para adaptarse a nuevos retos y desafíos producto de la interacción

entre distintos agentes económicos a nivel mundial. La educación es una institución que se ve afectada sensiblemente ante esta avanzada global. La liberalización e integración económica se acelera de manera dramática en la década de los noventa y genera impactos significativos en ámbitos de orden político, económico, social y cultural. Así, la educación adquiere un papel estratégico en diferentes países al reconocer la importancia de fomentar la instrucción académica, que genere actitudes racionales para impulsar el pensamiento económico acorde con los avances tecnológicos.

No se trata solo de la homologación y adecuación educativa entre sistemas educativos de los países, sino también la visión del mundo que las nuevas generaciones tendrán frente a los desafíos en todas las áreas del actuar humano. Es un cambio ante nuevas necesidades globales que comenzó a vislumbrarse desde principios de la década de los años sesenta con la corriente económica keynesiana, que exponía que el crecimiento de un país se originaba en gran medida por el desarrollo del capital humano (Becker, 1993), y posteriormente por la visión del desarrollo tecnológico (motivado por la investigación) conforme a los modelos de crecimiento de Solow (1957).

En cuanto a la educación básica, se reconoce su prioridad para el desarrollo económico y social de un país en el entendido de que la educación presenta una tasa de retorno que incide en reducir las desigualdades de una generación a otra (Becker, 2002). Además, desde la perspectiva funcionalista de Durkheim (1991), la función de la educación es vista como la transmisión de valores de una generación a otra, que no se da solo en el plano intelectual, sino también en la cotidianidad de un ancestro a un sucesor.

En este sentido, la formación económica-financiera en las instituciones de educación primaria puede ser necesaria para dotar de herramientas que permitan a los infantes comprender y enfrentar el entorno económico y social donde se desenvuelven y brindar instrumentos que subsanen fronteras de desigualdad.

Ahora bien, en términos de cambios en la educación, Dussel (1997) sostiene que el currículo académico es una propuesta cultural expresada en contenidos temáticos que en sí misma define elementos sociales y económicos de un país. Con base en ello, algunos países desarrollados comenzaron a adecuar sus sistemas educativos con aprendizajes y prácticas orientadas a la conformación de un individuo global al incluir contenidos curriculares propiamente relacionados con el entorno económico y financiero. Un caso particular es Japón, el cual, a partir de 1989, consideró la inclusión de temas económicos en la educación básica como un mecanismo de adaptación a la nueva economía global (Yamane, 1996). También en escuelas de Estados Unidos e Inglaterra se incorporó a la agenda educacional la formación de habilidades económicas y financieras en el esquema de educación básica (Yamane, 1997).

En cambio, en México, con las primeras manifestaciones del liberalismo económico, no se identifican cambios en la forma de concebir la educación. Con procesos de disolución del modelo de sustitución de importaciones en la década de los setenta, las crisis petroleras y financieras en los ochenta, y las crisis financieras internacionales de los noventa, no existió ese margen de adaptación del sistema educativo que ya era desarrollado en otras latitudes. 
Sin embargo, partimos de la idea de que el proceso de cambio en la educación básica de México inició con una reflexión de la Conferencia Mundial de la Educación para Todos (Tailandia, 1990), donde se asignó a la educación básica la responsabilidad de iniciar con la formulación de dos tipos de competencias genéricas que la Unión Europea las llamó competencias clave: la primera para la vida social y personal, y la segunda se refería a las académicas (Barriga, 2006). Esto no significa implementar un modelo educativo por competencias en México, pero sí reconocer el papel de la educación básica en el desarrollo humano.

En este orden de ideas, y como resultado de contingencias políticas, económicas, sociales y culturales, se generaron las primeras iniciativas del cambio en la educación básica a partir de la década de los noventa, al mostrar interés por la evaluación de la educación primaria, que tiene que ver con las pruebas estandarizadas y las evaluaciones internacionales (Martínez y Blanco, 2010).

Después de una serie de cambios en el modelo educativo, recientemente ha aparecido la inclusión temática específica sobre educación financiera, con la aprobación del modelo educativo en el nivel básico por parte de la Secretaría de Educación Pública (SEP, 2017). Con esta reforma se busca transitar de un sistema memorístico a uno que dé prioridad a la comprensión. Sin embargo, la educación financiera, entre otras competencias que se pretende desarrollar en los infantes, se propone de manera optativa (SEP, 2017).

La reforma en el modelo educativo de educación básica se entiende como la manifestación de un cambio de las formas en que el país procura satisfacer plenamente requerimientos sociales de actualidad. Por lo anterior, nuestro trabajo analiza la situación actual del conocimiento sobre temas económicos que tiene la población infantil de la zona metropolitana de Guadalajara (ZMG) con la intención de explorar el nivel de entendimiento económico del niño sobre el cual la reforma educativa va a impactar.

Tomamos en consideración el trabajo de Denegri, Gempp, Del Valle, Etchebarne y González (2006), quienes sostienen que la mayor comprensión y el desarrollo de una conceptualización económica básica se manifiestan entre los once y catorce años de edad. Con base en ello, pretendemos identificar los factores que inciden en el proceso de educación financiera en la vida cotidiana de los niños de sexto año de primaria de la ZMG, Jalisco, México. Esto, con el propósito de presentar una evidencia empírica de la forma en que la población infantil hace propio el conocimiento económico-financiero ante la ausencia temática del actual plan de estudios, y sopesar el impacto que podría tener una reforma aprobada con un enfoque más explícito de educación económica-financiera.

\section{REFERENTE TEÓRICO}

La educación económica ayuda a comprender y actuar de manera informada en las relaciones e intercambios entre el individuo y las actividades productivas. DiezMartínez (2009), al analizar la alfabetización en materia económica y financiera en México, la define como "una herramienta que permite enseñar a los niños y jóvenes a valorar las decisiones y consecuencias de su conducta y la conducta de otros en un amplio rango de cuestiones" (p. 8). 
Por su parte, Denegri et al. (2014) definen la educación económica como "la acción educativa intencionada, cuyo objetivo es aportar las nociones económicas básicas y las estrategias para tomar decisiones de consumo como personas conscientes, críticas, responsables y solidarias" (p. 84). En este orden de ideas, pensaríamos en la alfabetización financiera como un proceso en el seno familiar y la educación financiera de manera formalizada en el ámbito educativo, términos que en este artículo se plantean indistintamente con el propósito de valorar el resultado de la apropiación de conocimiento en materia económica-financiera.

La literatura sobre la educación financiera en niños y adolescentes puede tener dos perspectivas. La primera la encontramos en los estudios que describen los factores que influyen en la comprensión de niños y adolescentes sobre temas económico-financieros. Un ejemplo es el de Amar, Abello, Llanos y Gómez (2005), quienes señalan los elementos de educación financiera que se presentan en la familia y se apoyan en una investigación mixta. Al analizar a 49 familias colombianas, concluyen que las prácticas y estrategias que utilizan los padres de familia, de los estratos altos y bajos, se implementan con objetivos diferentes. Por una parte, los padres de familias de estrato bajo manifiestan que no es conveniente que "los niños tengan un manejo del dinero porque podría ser utilizado en cosas perjudiciales" (p. 60). Esta afirmación se da porque en el estrato bajo la atención se centra en el cuidado de los recursos del hogar. Por otra, las familias de estrato alto tratan de dejar una enseñanza más amplia y duradera en los hijos, y permiten que estos administren dinero desde temprana edad al otorgarles una mesada periódica y constante.

En un estudio más reciente, Llanos y Abello (2014) identifican la influencia de los padres en la educación financiera de sus hijos e hijas mediante una encuesta aplicada entre 2013 y 2014 en escuelas primarias de Colombia. Los autores concluyen que las estrategias de ahorro, de administración del dinero y estímulo de conductas económicas que brindan los padres de familia pueden influir en el desarrollo educativo financiero de sus hijos e hijas cuando se enseñan en constante interacción con la vida cotidiana.

Desde la misma perspectiva, pero enfocándose en factores contextuales exógenos como el sistema bancario, Denegri, Martínez y Etchebarne (2007) describen el proceso de la comprensión del funcionamiento bancario en adolescentes chilenos mediante un estudio mixto basado en un análisis de regresión y entrevistas en profundidad a 159 estudiantes de catorce a dieciocho años en distintas zonas económicas en Chile, entre 2005 y 2006. Los autores concluyen que se tiene una visión muy limitada de las funciones de un banco, ya que los informantes atribuyen mayor responsabilidad financiera al gobierno que a los individuos.

La segunda perspectiva se centra en aspectos intrapersonales de la comprensión de temas económicos en niños y adolescentes; aquí identificamos la perspectiva de la psicología evolutiva. Al respecto, Denegri, Del Valle, Gempp y Lara (2006) destacan la socialización económica en el seno familiar, donde se gesta lo relativo a la comprensión de la economía, y hacen hincapié en los estudios latinoamericanos. Asimismo, identifican tres etapas evolutivas de comprensión de temas económicos financieros que inician en la infancia: primero, la parte conceptual; segundo, la reflexión; y tercero, la aplicación y resolución de problemas. 
Estos autores concluyen que la formación económica formal podría implementarse exitosamente de los diez a los doce años, porque a esa edad ya cuentan con estructuras cognitivas operacionales para relacionar conceptos y las matemáticas con la cotidianidad, lo que contribuye al desarrollo de destrezas en el uso y administración del dinero, de planificación, toma de decisiones y resolución de problemas. También, esquematizan esta secuencia evolutiva en contenidos temáticos dentro de su propuesta "Yo y la economía" en Chile, y consideran la participación activa de los padres y los medios de comunicación en la enseñanza de los infantes.

Denegri et al. (2006) revisaron aspectos críticos y desafíos que son necesarios para plantear una propuesta de educación económica en la enseñanza básica, en virtud de que la formación universitaria en esa materia es insuficiente para garantizar conductas económicas eficientes, dado que los padres no siempre son acreedores de una adecuada formación económica-financiera. A partir de una extensa revisión de literatura sobre la comprensión del mundo económico, estos autores presentan el modelo de psicogénesis del pensamiento económico desarrollado en 1995, e identifican una secuencia evolutiva que consiste en el desarrollo progresivo de herramientas cognitivas disponibles a partir de la infancia que propician el acceso a experiencias de manejo de dinero, las cuales permiten la comprensión de conceptos económicos desde temprana edad. Los mismos autores identifican que, a partir de los once años, el niño posee estructuras cognitivas para relacionar e integrar bases matemáticas con conceptos y eventos de un mundo económico.

De manera similar, Denegri et al. (2014) encuentran brechas de conocimiento en educación financiera y económica a partir de investigaciones de carácter cualitativo en diferentes ámbitos de la sociedad chilena de 2003 a 2014, donde se determina que deben existir intervenciones centradas en la población adulta del sector socioeconómico bajo y medio, intervenciones con enfoque de género, en el segmento de preadolescentes y adolescentes, así como de profesores y estudiantes de pedagogía. Además, se propone un modelo educativo para los temas de educación económica y financiera en la formación inicial docente.

En otros casos, esta perspectiva también ha proporcionado la información y argumentación suficiente para trabajos como los de Diez-Martínez (2009), quien presenta algunas reflexiones sobre las definiciones y los alcances de la educación financiera, y emprende una serie de investigaciones en distintas escuelas en México de 1995 a 2008; propone que se aborde el tema económico en la educación básica desde una perspectiva psicológica evolutiva.

A partir del reconocimiento de que en México se ha dejado la educación financiera a las instituciones fiduciarias, Diez-Martínez (2016) analiza la comprensión de algunas variables económicas como el trabajo, la remuneración y los intereses en los préstamos bancarios a una población de adolescentes estudiantes mexicanos entre doce y dieciséis años de edad. Con los resultados de una encuesta, la autora realiza un análisis descriptivo y, a partir de la psicología del desarrollo del pensamiento económico, concluye que se tiene muy poco entendimiento de conceptos económicos, lo que demuestra el bajo nivel de cultura financiera que tiene la población.

Después de haber revisado las dos perspectivas, nos damos cuenta de la precariedad de la educación financiera en los contenidos de educación básica en los países considerados en esta revisión: México, Colombia y Chile. Por ello, la única fuente de 
información financiera para la población infantil y adolescente es la familia. Esta ausencia de aprendizaje escolar tiene efectos y consecuencias en los niveles de entendimiento económico-financiero de los menores, lo cual tiene impacto en su actuación como agentes económicos en su ámbito cotidiano presente y futuro. Esta situación, como país, nos coloca en desventaja con naciones desarrolladas, como Japón, Inglaterra y Estados Unidos, que sí abonan al entendimiento de una economía global desde la educación básica.

Este entendimiento económico-financiero en la infancia da sentido a la educación financiera de acuerdo con la definición de Yamane (1996), quien refiere que la educación financiera se da en el campo de los sentidos, donde se busca sensibilizar al infante en el conocimiento económico de los sectores productivos de un país a fin de comprender el funcionamiento de ese sector, lo que puede generar una enseñanza y la reflexión sobre los usos y el cuidado de los recursos.

Por otra parte, además de la realidad latinoamericana expuesta por los autores referidos en párrafos anteriores, McCormick (2009) identifica que se requiere un plan de acción para integrar educación financiera en el currículo académico de los niños, ya que esto puede impactar el comportamiento de herramientas de ahorro. Sin embargo, este autor asume que un curso en la educación básica sería inadecuado, ya que primero es necesario brindar algunas ideas e información económicafinanciera a los niños.

Teniendo en cuenta la literatura sobre el tema, en este trabajo nos apoyamos en la perspectiva teórica de Bruner (1969), con sus consideraciones sobre psicología evolutiva, quien postula la teoría de la instrucción mediante las reglas respecto al modo más eficaz de lograr conocimientos y destrezas; también formula criterios y condiciones para satisfacerlos. Los pilares que soportan esta teoría son: la predisposición para aprender, la estructura del conocimiento, la secuencia y el reforzamiento. Estos son los elementos que utilizaremos para identificar la adquisición del conocimiento y conductas que adquieren los niños en materia de economía financiera, en el entendido de que el niño es un participante de la familia en un proceso amplio en el cual se negocian significados. En este orden de ideas, Bruner (1988) sostiene que el constructivismo de la psicología cultural es una expresión profunda que exige que nos hagamos conscientes y responsables de cómo conocemos y por qué; sin embargo, no pretende que haya una sola forma de construir el significado.

Para nuestro estudio, asumimos que el manejo cognitivo de un mundo económico en el niño se adquiere por medio de estrategias tendentes a reducir la complejidad y la confusión; por ello, consideramos las situaciones que "interesan" a los infantes en materia económica de la cotidianidad, porque, de acuerdo con Bruner (2006), existe una interacción constante entre la experiencia y la memoria en la construcción de significados y, para el caso que nos ocupa, las experiencias económicas corresponden a lo cotidiano.

\section{Metodología}

Nuestra investigación se aborda desde el paradigma positivista y el análisis es exploratorio descriptivo. Para tal fin, aplicamos una encuesta mediante un cuestionario que se construyó tomando en consideración los postulados de la teoría de la 
instrucción de Bruner, en términos de la predisposición para aprender, la estructura del conocimiento, la secuencia y el reforzamiento. Planteamos 25 preguntas, en su mayoría de opción múltiple, con el propósito de que fueran accesibles a los niños; también incluimos algunas respuestas abiertas para identificar las ideas que subyacen en la información que tienen los niños acerca de sus observaciones y el conocimiento relativo al ahorro, la forma de gastar y la función de un banco.

Estas respuestas abiertas se clasificaron, posteriormente, en categóricas para llevar a cabo el análisis de la información y buscar la relación entre variables. Para el tratamiento de la información, utilizamos el Statistical Package for the Social Sciences (SPSS). Ahora bien, para evaluar la predisposición a aprender, la estructura de conocimiento, secuencia y búsqueda de recompensas por parte del niño, elaboramos tablas de contingencia con la prueba estadística chi-cuadrada.

Para aplicar la encuesta, acudimos en forma directa a cinco primarias de la ZMG. La selección corresponde al intento de tener una cobertura del área geográfica. Administramos el cuestionario en papel para cada infante, con las indicaciones de las preguntas, y permanecimos en el aula para cualquier duda que tuvieran los niños. Esta actividad se realizó en mayo de 2017 en los domicilios de las escuelas.

La unidad de observación fueron los estudiantes de sexto grado en cinco escuelas públicas ubicadas en diferentes zonas geográficas con diverso contexto económico y social; al final, obtuvimos una muestra de 244 niños entre once y catorce años (ver tabla 1).

Tabla 1. Especificación de la muestra de estudio

\begin{tabular}{|c|c|c|c|c|c|c|c|}
\hline \multirow{2}{*}{ Escuela } & \multicolumn{4}{|c|}{ Género } & \multicolumn{2}{c|}{ Total } & \multirow{2}{*}{ Municipio } \\
\cline { 2 - 7 } & \multicolumn{2}{|c|}{ Niñas } & \multicolumn{2}{|c|}{ Niños } & Absoluto & Porcentaje & Tonalá \\
\hline Escuela 1 & 16 & $46 \%$ & 19 & $54 \%$ & 35 & 14 & Tonalá \\
\hline Escuela 2 & 26 & $49 \%$ & 28 & $51 \%$ & 54 & 22 & Zapopan \\
\hline Escuela 3 & 40 & $47 \%$ & 45 & $53 \%$ & 85 & 35 & Guadalajara \\
\hline Escuela 4 & 23 & $42 \%$ & 32 & $58 \%$ & 55 & 23 & Tlaquepaque \\
\hline Escuela 5 & 8 & $53 \%$ & 7 & $47 \%$ & 15 & 6 & 100 \\
\hline Total & 113 & $47 \%$ & 131 & $53 \%$ & 244 & & \\
\hline
\end{tabular}

\section{RESULTADOS}

En el análisis descriptivo, encontramos que la edad de los niños de sexto año de primaria oscila entre los once y doce años, y en el rango superior, $2 \%$ tienen entre catorce y dieciséis años. En relación con los ingresos que ellos administran de manera libre, esto es, lo que sus padres les dan en promedio diario o semanalmente, los datos globales nos arrojan un promedio de 76 pesos por semana. Identificamos también que los niños reciben el gasto semanal promedio diferente en cada municipio de la ZMG. Debemos mencionar que, del total, 7\% de los niños no reciben dinero para gasto diario o semanal por parte de sus padres. En este aspecto contrasta Zapopan, un municipio donde se presentan casos extremos: niños que reciben alrededor de cien pesos o más por semana y niños que no reciben nada para su gasto diario. 
El análisis de la información se realizó buscando relaciones entre variables que denoten el modo en que los niños adquieren y hacen uso del conocimiento económico financiero en la cotidianidad. Para este fin, metodológicamente formulamos hipótesis que corresponden a pruebas de independencia chi-cuadrada, con nivel de significancia $\alpha=0.05$.

Las variables que contrastamos en estas pruebas son categóricas; en este caso, identificamos las frecuencias de las diferentes categorías consideradas en la encuesta. El interés es probar una supuesta independencia de comportamientos, conocimientos o percepciones de los niños al comparar lo que observan de sus padres y las acciones que refieren comportamientos económico-financieros. Para este fin, formulamos una serie de hipótesis:

-Hipótesis nula: El hecho de que el niño se fije en precios es independiente de que observe a sus padres comparar precios al realizar el súper de la casa.

Tabla de contingencia 2. ¿Los padres de familia comparan precios al realizar las compras del hogar? *Curiosidad del niño por fijarse en precios

\begin{tabular}{|c|c|c|c|c|c|c|}
\hline & & & & \multicolumn{2}{|c|}{ Niños identifican precios } & \multirow{2}{*}{ Total } \\
\hline & & & & No & Sí & \\
\hline \multirow{2}{*}{ Papás identifican precios } & No & \multicolumn{2}{|c|}{ Recuento } & 4 & 16 & 20 \\
\hline & Sí & \multicolumn{2}{|c|}{ Recuento } & 32 & 192 & 224 \\
\hline \multirow[t]{2}{*}{ Total } & & \multicolumn{2}{|c|}{ Recuento } & 36 & 208 & 244 \\
\hline & & Valor & df & \multicolumn{3}{|c|}{ Significación asintótica (bilateral) } \\
\hline Chi-cuadrado de Pearson & & $73.833^{a}$ & 5 & \multicolumn{3}{|c|}{.000} \\
\hline
\end{tabular}

Como advertimos en la tabla 2, la hipótesis nula es rechazada: la significancia de la prueba $(\mathrm{P}=0.000 \leq 0.05)$, siendo inferior al valor de $\alpha(0.05)$, revela que las enseñanzas acerca de los precios se perciben como una acción observada de sus padres.

Así también, respecto al actuar del niño en un contexto de toma de decisión de compras a su alcance: golosinas, a partir del presupuesto que sus padres le otorgan, se plantea la siguiente hipótesis:

-Hipótesis nula: El hecho de que el niño se fije en precios es independiente de sus preferencias entre sabor, precio y cantidad a la hora de comprar.

Tabla de contingencia 3. Curiosidad del niño por identificar precios.

*Lo más importante al comprar

\begin{tabular}{|c|c|c|c|c|c|c|c|}
\hline & & & & Sabor & Precio & Cantidad & Total \\
\hline \multirow[t]{2}{*}{ Identificar Precios } & No & & Recuento & 22 & 5 & 9 & 36 \\
\hline & Sí & & Recuento & 100 & 79 & 29 & 208 \\
\hline \multirow{2}{*}{\multicolumn{2}{|c|}{ Total }} & & Recuento & 122 & 84 & 38 & 244 \\
\hline & & Valor & $\mathrm{df}$ & \multicolumn{4}{|c|}{ Significación asintótica (bilateral) } \\
\hline \multicolumn{2}{|c|}{ Chi-cuadrado de Pearson } & $8.626^{a}$ & 2 & \multicolumn{4}{|c|}{.013} \\
\hline
\end{tabular}


Nuevamente, rechazamos la hipótesis nula. Encontramos evidencia estadística de que el fijarse en los precios está relacionado con sus preferencias, lo que le ayuda a tomar decisiones de compra: lo más importante es el sabor y, en segundo lugar, el precio del producto al comprar. Esto, según las frecuencias observadas en la tabla de contingencia 3: 122 de 244 niños refieren el sabor como el elemento principal al decidir comprar golosinas.

Esta relación nos brinda pautas para identificar la secuencia del aprendizaje económico-financiero de acuerdo con Bruner (1969), quien refiere la especificación del orden que el niño brinda a la observación y la actuación personal. Ahora bien, esa atención del niño hacia los precios en su relación con la experiencia de acompañar a los padres (quienes son los tomadores de decisiones) de manera cotidiana, nos ayuda a generar la siguiente hipótesis:

-Hipótesis nula: El hecho de que el niño se fije en precios es independiente del gusto por acompañar a sus padres en las compras.

Tabla de contingencia 4. Curiosidad del niño por identificar precios. ${ }^{*}$ ¿Te gusta acompañar a tus papás a las compras?

\begin{tabular}{cccc|c|c|c} 
& & & No & A veces & Sí & Total \\
\hline Identificar precios & No & Recuento & 5 & 18 & 13 & 36 \\
\cline { 2 - 8 } & Sí & Recuento & 11 & 79 & 118 & 208 \\
\hline Total & & Recuento & 16 & 97 & 131 & 244 \\
\hline Chi-cuadrado de Pearson & Valor & df & \multicolumn{3}{c}{ Significación asintótica (bilateral) } \\
\hline
\end{tabular}

El rechazo de la hipótesis nula hace evidente la predisposición para aprender, al mostrar interés en las actividades cotidianas de los padres. Al darnos cuenta de que sí es relevante para el infante acompañar a sus padres en las compras, e indagar el motivo, planteamos la siguiente hipótesis:

-Hipótesis nula: El hecho de que el niño se fije en precios es independiente del motivo por el cual acompaña a sus padres en las compras.

Tabla de contingencia 5. Curiosidad del niño por identificar precios.

*Motivo para acompañar a papás a compras

\begin{tabular}{lcc|c|c|c|c} 
& & Interés & $\begin{array}{c}\text { La experiencia } \\
\text { de comprar }\end{array}$ & $\begin{array}{c}\text { Ayudar a } \\
\text { mis papás }\end{array}$ & $\begin{array}{c}\text { Me aburro, } \\
\text { me da flojera }\end{array}$ & Total \\
\hline Identificar precios & No & 11 & 6 & 4 & 15 & 36 \\
\hline Sí & 81 & 63 & 25 & 39 & 208 \\
\hline Total & 92 & 69 & 29 & 54 & 244 \\
\hline \multicolumn{2}{l|}{} & Valor & df & \multicolumn{2}{|c}{ Significación asintótica (bilateral) } \\
\hline Chi-cuadrado de Pearson & $9.890^{a}$ & 3 & \multicolumn{3}{|c}{.020} \\
\hline
\end{tabular}

a. 1 casillas (12.5\%) han esperado un recuento menor que 5. El recuento mínimo esperado es 4.28.

El rechazo de la hipótesis nula nos da evidencia de que no es independiente el fijarse en los precios y el motivo para acompañar a los padres en las compras. Esta prueba nos sugiere la posibilidad de que el niño vive, en general, una expe- 
riencia satisfactoria al participar en las actividades de compras con sus padres. La tabla de contingencia 5 engloba intereses específicos, como que los papás les compren "algo", "es divertido tener el carrito del súper" y "ver juguetes". Este es el resultado de una pregunta abierta acerca de los motivos que tienen los infantes para acompañar a sus padres en las compras, y que todo ha sido incluido en el apartado de interés.

En relación con el ahorro, con base en lo que los infantes identifican en las prácticas de sus padres, formulamos la siguiente hipótesis:

-Hipótesis nula: El hecho de que el niño ahorre es independiente del conocimiento que tiene de que sus padres también ahorran.

Tabla de contingencia 6. ¿Sabes si tus papás ahorran? ${ }^{*}$ ¿Tú ahorras?

\begin{tabular}{llc|c|c} 
& & \multicolumn{2}{c}{ Total } \\
\hline Sabes si tus papás ahorran & No ahorran & 2 & 4 & 6 \\
\cline { 2 - 6 } & No sé & 23 & 34 & 57 \\
\cline { 2 - 6 } & Sí ahorran & 31 & 150 & 181 \\
\hline Total & & 56 & 188 & 244 \\
\hline Chi-cuadrado de Pearson & Valor & df & Significación asintótica (bilateral) \\
\hline
\end{tabular}

Al rechazar la hipótesis nula, de acuerdo con la tabla de contingencia 6 (chi-cuadrado $=13.596 ; \mathrm{P}=0.001$ ) advertimos que los niños tienen presente el concepto de ahorro como una estructura del conocimiento que hacen propio al momento que ellos deciden ahorrar. Aunado a esto, 57 niños no tienen conocimiento del comportamiento de sus padres hacia el ahorro, que es cercano al número de niños que no ahorran (56). No obstante, 31 niños saben que sus padres sí ahorran $\mathrm{y}$ ellos no lo hacen.

En relación con el conocimiento de una estructura financiera institucional formal como son los bancos, ante las variables que se contrastan a continuación, se formula la hipótesis:

-Hipótesis nula: El hecho de que el niño identifique dos alternativas para pedir dinero prestado es independiente de los motivos por los cuales reconoce a la familia y al banco como las mejores opciones.

El planteamiento de esta hipótesis se construye con la base de la estructura de conocimiento de los niños. Al responder libremente la razón por la cual les parece mejor pedir a la familia o al banco, ellos mencionan que es por cobro de intereses, seguridad, confiabilidad, presión y evitar problemas, como se expone en la tabla de contingencia 7. 
Tabla de contingencia 7. ¿Cuál es la mejor opción para pedir prestado?

*¿Cuál es la razón para pedir a familiares o banco?

\begin{tabular}{cc|c|c|c|c|c|c} 
& \multicolumn{9}{c}{ Motivos } \\
& \multicolumn{1}{c|}{$\begin{array}{c}\text { No } \\
\text { sé }\end{array}$} & $\begin{array}{c}\text { Es más } \\
\text { seguro }\end{array}$ & $\begin{array}{c}\text { Son más } \\
\text { confiables }\end{array}$ & $\begin{array}{c}\text { No te } \\
\text { presionan }\end{array}$ & $\begin{array}{c}\text { Evitar } \\
\text { problemas }\end{array}$ & $\begin{array}{c}\text { El banco } \\
\text { cobra } \\
\text { intereses }\end{array}$ & Total \\
\hline Familiares & 9 & 16 & 76 & 22 & 3 & 12 & 138 \\
\hline Banco & 6 & 54 & 13 & 21 & 10 & 2 & 106 \\
\hline Total & 15 & 70 & 89 & 43 & 13 & 14 & 244 \\
\hline & & \multicolumn{2}{|c|}{ Valor } & df & Significación asintótica (bilateral) \\
\hline Chi-cuadrado de Pearson & \multicolumn{2}{|c|}{$73.833^{a}$} & 5 & & .000 \\
\hline
\end{tabular}

El rechazo de la hipótesis nula refiere que en el ámbito de conocimiento del infante existe una dependencia entre lo que el niño percibe como mejor opción: al banco por ser más seguro y a la familia, por ser de mayor confiabilidad. De acuerdo con la teoría de la instrucción de Bruner, el infante genera una estructura de conocimiento. En nuestro caso de estudio, 76 niños refieren que los familiares son más confiables para pedir prestado y 54 niños identifican al banco como una opción más segura para un préstamo. Estas visiones sobre la función del banco, según su percepción y significado familiar, son acordes con su nivel conceptual y de entendimiento vivencial afectivo.

En el entendido de que los niños tienen una estructura de conocimiento en relación con el banco, resulta importante indagar quién o en qué ambiente se genera esta estructura. Por tanto, se formula la siguiente hipótesis:

-Hipótesis nula: El conocimiento sobre la utilidad de un banco es independiente de la(s) persona(s) que le brindaron la información.

La tabla de contingencia 8 nos muestra que, de 244 niños, solo siete no tienen conocimiento de la utilidad de un banco; sin embargo, es evidente que estos obtienen su estructura de conocimiento en gran medida de sus papas; en cambio, los maestros alcanzan una mínima proporción que denota la ausencia de información sobre temas económicos en la escuela. Por tal motivo, consideramos que la reforma educativa puede impactar positivamente en la educación financiera de los niños.

Tabla de contingencia 8. ¿Para qué sirve un banco?

*¿Quién te explicó?

\begin{tabular}{|c|c|c|c|c|c|}
\hline \multicolumn{3}{|c|}{ Explicó } & Maestros & Papás & Total \\
\hline \multirow[t]{6}{*}{ Utilidad de un banco } & No sé & & 2 & 5 & 7 \\
\hline & Ahorrar & & 11 & 100 & 111 \\
\hline & Retirar o pedir prés & amo & 0 & 44 & 44 \\
\hline & Depósito, ahorro y c & édito & 6 & 76 & 82 \\
\hline & & Total & 19 & 225 & 244 \\
\hline & Valor & df & \multicolumn{3}{|c|}{ Significación asintótica (bilateral) } \\
\hline Chi-cuadrado de Pearson & $8.649^{a}$ & 3 & \multicolumn{3}{|c|}{.034} \\
\hline
\end{tabular}


En este caso, el rechazo de la hipótesis nula queda inconcluso debido a esa pequeña proporción de niños que no saben qué es un banco. Sin embargo, si formulamos de nuevo la hipótesis excluyendo a quienes no saben para qué sirve un banco, nuestra evidencia es contundente para afirmar que sí existe una relación de dependencia entre conocimiento y personas que brindan información a los niños.

La predisposición para aprender en los niños queda patente en cualquier ambiente, sea familiar o escolar. En este sentido, la escuela es un espacio donde el niño puede significar lo que percibe en su ámbito familiar relacionado con las finanzas personales. Por naturaleza, el niño tiene curiosidad por conocer las cosas que lo rodean. La escuela puede contribuir en su aprendizaje económico relacionando experiencias con una instrucción mediada y guiada por el maestro.

En su mayoría, el niño recibe dinero de sus padres a diario; es deseable que sea la misma cantidad para que el niño pueda ajustarse a la capacidad de gastar y pensar en ahorrar. Esto coincide con el estudio de Llanos y Abello (2014), quienes destacan la importancia de inducir al niño a administrar sus recursos.

La predisposición para aprender es evidente en las respuestas de los niños al prestar atención a lo que hacen sus padres en la vida cotidiana, como es el caso de comparar precios al momento de realizar compras. La estructura del conocimiento es la manera en que se presenta este; en este caso, se trata de la utilidad de un banco; además, resulta importante identificar que, principalmente, esta estructura se genera en el ambiente familiar y se simplifican las observaciones del entorno familiar para generar nuevas proposiciones de comportamiento propio; con ello se manifiesta la capacidad de observación y aprendizaje, y se reconoce que la naturaleza no es absoluta, sino relativa.

La secuencia del conocimiento es la especificación del orden que el niño brinda a la observación y la actuación de los entes económicos; esto es, el niño ahorra porque aprende de sus padres. Finalmente, el reforzamiento dentro de la teoría de la instrucción refiere la naturaleza y frecuencia de las recompensas; en nuestra investigación se manifiesta en el interés en que sus padres le compren algo o vivir una experiencia agradable al participar en las compras del hogar.

\section{CONCLUSIONES}

A partir de las características principales de la teoría de la instrucción de Bruner (1969), en esta investigación damos cuenta de un aprendizaje, conocimiento o habilidad económico-financiera en los niños. El común denominador de las escuelas analizadas en la ZMG, y las escuelas de otros países latinoamericanos como Chile y Colombia, es la falta de una formación económica financiera en la educación básica, aun cuando se reconoce que la infancia es la edad óptima para iniciar este tipo de competencias para la vida.

De acuerdo con los resultados de nuestra investigación, el principal factor que incide en la alfabetización financiera para el desarrollo de destrezas y habilidades en los niños es la influencia del ambiente familiar. Mediante la observación constante 
de los infantes en prácticas cotidianas, como acompañar a sus padres en las compras o el fijarse en los precios, estos muestran una disposición a aprender sobre la actividad económica-financiera del entorno familiar.

En términos de ahorro, es evidente que el infante que ahorra es quien se da cuenta de que sus padres también lo hacen; asimismo, al reconocer que una de las dos mejores opciones para pedir prestado es el banco, por ser más seguro, y la otra es la familia, por ser más confiable. Aunque lo anterior es una señal de la carencia de información del infante ante su visión sesgada por el entorno familiar, revela del mismo modo que el niño no desconoce la función del banco -gracias a la experiencia de sus padres-, lo que constituye un primer patrón de construcción conceptual del pensamiento económico.

Por tanto, la propuesta educativa en el nivel de primaria podría iniciar a partir del supuesto de la psicogénesis del pensamiento, esto es, el niño posee un conocimiento previo del mundo económico. Además, la información proporcionada al niño se puede ampliar en un ámbito macroeconómico, es decir, al interior de la institución educativa a fin de generar en el infante una conceptualización y visión más amplia al relacionar impactos de comportamiento individual en un contexto global; por ejemplo, dar nociones de consumo y gasto en un país, el valor económico de los recursos naturales, y cómo surgen las crisis financieras en el mundo. Aún más, países como Japón, Estados Unidos e Inglaterra consideran que la información económica genera conciencia de la economía y de los recursos de su país. En este sentido, pensar en la educación sin vincularla a la contribución del desarrollo del país sería un modelo inacabado.

Aunado a la parte empírica, nuestro trabajo explora los efectos que se infieren de las relaciones que guardan la observación y la acción de los infantes, en virtud de que la alfabetización económica inicia en el seno familiar. Por tanto, es importante darle seguimiento en la escuela, según la propia naturaleza del niño, para lograr en él un desarrollo en todas sus facetas, al integrar relaciones de contenidos y estrategias cognitivas para que observen y signifiquen un mundo económico global; esto, acorde con los postulados de la teoría de la instrucción de Bruner (1969).

Al hablar de una reforma en el ámbito educativo, la entendemos como un proceso de innovación en la educación, tanto en su currículo como en su operación. La evidencia empírica aquí mostrada nos habla de la necesidad de incluir la educación financiera en el contexto de reforma educativa como parte de un cambio en la manera en que se configura y modela la visión del infante acerca de los problemas económico-financieros contemporáneos. Este nuevo modo de incorporar el pensamiento financiero y económico en la educación primaria, de acuerdo con el modelo de psicogénesis del pensamiento económico, apoyará el proceso de reforma educativa que se busca, con niveles de escolaridad más altos y de mayor calidad, a fin de mejorar la condición económica y social en México.

Hay que tener en cuenta que el desarrollo del niño implica influencia mutua entre familia y escuela. El entorno social juega un papel relevante en el proceso enseñanza-aprendizaje; especialmente, en la población infantil es necesario considerar actividades significativas a su nivel de comprensión. Si bien es cierto que nada garantiza que el modelo educativo funcione y proporcione las herramientas necesarias para comprender la dinámica económica de un mundo global, que en un futuro 
impacte de manera positiva el desarrollo económico, dado que no es una formación obligatoria y depende del criterio de cada escuela, la formalización de la educación financiera en el contexto escolar ayudaría a coordinar las actividades rutinarias de enseñanza-aprendizaje que refieren el entendimiento y significado de temas económico-financieros vinculados al entorno familiar y social.

Vale la pena aclarar que los resultados de la investigación, aun cuando no se pueden hacer inferencias a escala nacional, por la limitante de la muestra específica de un área geográfica del estado de Jalisco, pueden ser un referente para indagar la forma en que los niños adquieren conocimiento económico en diferentes zonas del país. Asimismo, nuestros hallazgos se pueden aprovechar para formular estrategias en las escuelas que activen la predisposición a aprender y despierten la curiosidad del niño y su interés por temas económicos del mundo global. Con la intervención y guía de los maestros, basada en la reforma del modelo educativo, se puede lograr que la estructura del conocimiento avance a un nivel de información más amplio sobre temas económicos, que le permita al niño relacionar lo que vive en su hogar y los impactos en el mundo global en términos de ahorro, gasto e, incluso, recursos naturales.

\section{Agradecimientos}

A los directores y las maestras encargadas de los grupos de sexto año de las escuelas primarias de educación pública del turno matutino, cuyo apoyo fue invaluable en esta investigación. A Armando Fuentes Jaime, por su colaboración en la investigación, y a Cynthia Guadalupe Ramírez Álvarez, por su trabajo de campo y captura de datos.

\section{REFERENCIAS BIBLIOGRÁFICAS}

Amar Amar, J., Abello Llanos, R., Llanos, M. \& Gómez, B. (2005). Estrategias y prácticas socializadoras y de alfabetización económica en familias de una ciudad multifinanciera de la región caribe colombiana. Psicología desde el Caribe, núm. 16, pp. 29-63. Recuperado de http://rcientificas.uninorte.edu.co/ index.php/psicologia/article/view/1907

Barriga Díaz, A. (2006). El enfoque de competencias en la educación. ¿Una alternativa o un disfraz de cambio? Perfiles Educativos, vol. 28, núm. 111, pp. 7-36. Recuperado de www.iisue.unam.mx/perfiles/descargas/pdf/2006-111-7-36

Becker, G. S. (2002). La economía cotidiana. Ciudad de México: Editorial Planeta Mexicana.

Becker, G. S. (1993). Human capital. A theoretical and empirical analysis, with special reference to education (3a. ed.). Chicago, EUA: The University of Chicago Press.

Bruner, J. S. (1969). Hacia una teoría de la instrucción. Ciudad de México, México: Unión Tipográfica Editorial Hispano Americana.

Bruner, J. S. (1988). Desarrollo cognitivo y educación. Madrid, España: Ediciones Morata.

Bruner, J. S. (2006). Actos de significado. Madrid, España: Alianza Editorial.

Denegri, M., Del Valle Rojas, C., Gempp, R. y Lara Arzola, M. (2006). Educación económica en la escuela: hacia una propuesta de intervención. Estudios Pedagógicos, vol. 32, núm. 2, pp. 103-120. Recuperado de https://dialnet.unirioja. es/servlet/articulo?codigo $=2269613$ 
Denegri, M., Del Valle Rojas, C., González, Y., Etchebarne, S., Sepúlveda A. J. y Sandoval G. D. (2014). ¿Consumidores o ciudadanos? Una propuesta de inserción de la educación económica y financiera en la formación inicial docente. Estudios Pedagógicos, vol. 40, núm. 1, pp. 75-96. Recuperado de http:// go.galegroup.com/ps/anonymous?id=GALE\%7CA466167121\&sid=googleSc holar\&v=2.1\&it=r\&linkaccess $=a b s \& i s s n=0716050 X \& p=A O N E \& s w=w$

Denegri, M., Gempp, R., Del Valle, C., Etchebarne, S. y González, Y. (2006). El aporte de la psicología educacional a las propuestas de educación económica: los temas claves. Revista de Psicología, vol. 15, núm. 2, pp. 77-94. Recuperado de https://revistas.uchile.cl/index.php/RDP/article/view/18398

Denegri, M., Martínez, G. \& Etchebarne, S. (2007). La comprensión del funcionamiento bancario en adolescentes chilenos: un estudio de psicología económica. Interdisciplinaria, vol. 24, núm. 2, pp. 137-159. Recuperado de http:// go.galegroup.com/ps/anonymous?id=GALE\%7CA182979188\&sid=googleSc holar\&v=2.1\&it=r\&linkaccess=abs\&issn=03258203\&p=AONE\&sw =w

Diez-Martínez, E. (2016). Alfabetización socioeconómica y financiera en adolescentes mexicanos del siglo XXI. Revista Electrónica de Investigación Educativa, vol. 18, núm. 2, pp. 130-143. Recuperado de http://redie.uabc.mx/redie/article/view/861

Diez-Martínez, E. (2009). La alfabetización socioeconómica y financiera y la educación para el consumo sostenible en México: algunas reflexiones desde la psicología y la educación. CPU-e. Revista de Investigación Educativa, núm. 8, pp. 1-15. Recuperado de http://revistas.uv.mx/index.php/cpue/article/view/85

Durkheim, É. (1991). Educación y sociología (3ra. ed.). México: Editorial Colofón.

Dussel, I. (1997). Curriculum, humanismo y democracia en la enseñanza media (1863-1920). Buenos Aires: FLACSO.

Llanos Martínez, M. y Abello, R. (2014). Incidencia de las estrategias de alfabetización económica de los padres en el ámbito familiar sobre el desarrollo del pensamiento económico de los hijos. Universitas Psychologica, vol. 14, núm. 1, pp. 177-188. http://dx.doi.org/10.11144/Javeriana.upsy14-1.ieae

Martínez Rizo, F. y Blanco, E. (2010). La evaluación educativa. Experiencias, avances y desafíos. En Arnaut y Giourguli (eds.). Educación (vol. V, pp. 89-123). México: El Colegio de México, Colección Los grandes problemas de México.

McCormick Henn, M. (2009). The effectiveness of youth financial education: A review of the literature. Journal of Financial Counseling and Planning, núm. 20, vol. 1, pp. 70-83. Recuperado de https://ssrn.com/abstract=2225339

Secretaría de Educación Pública. (2017). Modelo educativo para la educación obligatoria. Ciudad de México.

Solow, R. M. (1957). Technical Change and the Aggregate Production Function. Review of Economics and Statisitics, núm. 39, pp. 312-320. Recuperado de https:// faculty.georgetown.edu/mh5/class/econ489/Solow-Growth-Accounting.pdf

Yamane, E. (1997).The meaning of "economics education" in Japanese Elementary and Secondary Education: An historical perspective. Presentado en la Second Conference of the International Asssociation for Children's Social and Economics Education. Malmö, Suecia, Edge Hill University College/IACSEE.

Yamane, E. (1996). Social and economic education in the Japanese Elementary School National Curriculum. Citizenship, Social and Economics Education, vol. 1, núm. 1, pp. 31-44. Recuperado de http://journals.sagepub.com/doi/ pdf/10.2304/csee.1996.1.1.31 